\title{
MODELAGEM 3D DE EDIFÍCIOS HISTÓRICOS: A INFLUÊNCIA DO LOD PROCESSO DE RECONSTRUÇÃO VIRTUAL
}

\section{D MODELING OF HISTORIC BUILDINGS: THE INFLUENCE OF LOD IN THE VIRTUAL RECONSTRUCTION PROCESS}

\author{
João Paulo Chagas Maia Vilela ${ }^{1}$, Ricardo Ferreira Lopes ${ }^{1}$, Fernando Lima ${ }^{1,2}$
}

\section{RESUMO:}

O presente artigo é fruto de uma pesquisa que investiga a modelagem tridimensional (3D) de edifícios históricos mediante diferentes configurações de LOD (Level Of Detail). No âmbito do patrimônio virtual, a criação de modelos 3D requer tempo, conhecimento técnico e sobretudo, planejamento. O LOD, neste caso, influencia na construção, representação e processamento dos modelos produzidos. Este estudo tem como objetivo principal investigar como o LOD interferiu no processo de reconstrução virtual de um edifício histórico localizado no município de Juiz de Fora, Minas Gerais, Brasil. A metodologia empregada valeu-se da combinação das pesquisas bibliográfica e experimental. Foi utilizada a técnica de modelagem semântica. A modelagem 3D do edifício foi desenvolvida em cinco diferentes LODs, cada qual atrelado às estruturas semânticas da arquitetura investigada. A partir disso, foram coletados quatro dados referentes à modelagem de cada LOD: tamanho do arquivo, tempo de modelagem, tempo de renderização e a quantidade de dados do modelo. Posteriormente, estes dados foram comparados. $O$ experimento demonstrou relação direta entre os fluxos de trabalho e os LODs dos modelos. Acredita-se que ao investigar o processo de modelagem 3D, é possível oferecer suporte a futuros trabalhos da área, bem como ampliar as discussões sobre questões teóricopráticas voltadas à construção de modelos 3D de edifícios históricos.

PALAVRAS-CHAVE: Patrimônio virtual; Modelagem 3D; Level Of Detail; Modelagem semântica.
Fonte de Financiamento: Coordenação de Aperfeiçoamento de Pessoal de Nível Superior.

Conflito de Interesse: Declara não haver.

Ética em Pesquisa: Declara não haver necessidade.

Submetido em: data de submissão: 01/09/2020 Aceito em: 24/03/2021

KEYWORDS: Virtual heritage; 3D modelling; Level Of Detail; Semantic modelling. 


\section{INTRODUÇÃO}

A crescente evolução das tecnologias digitais e o surgimento da realidade virtual (RV) trouxeram grandes avanços para diversas áreas do conhecimento. A possibilidade de construir virtualmente 'realidades' que 'imitem' o mundo real tornou-se uma ferramenta de grande potencial para simular cenários, visualizar objetos, prever resultados e, até mesmo, planejar tarefas (LÉVY, 2010). Neste contexto, as atividades voltadas ao reconhecimento, à proteção e à manutenção do patrimônio cultural têm cada vez mais se apoiado em ferramentas e plataformas digitais (ALENCAR, 2013).

0 patrimônio arquitetônico, tema de investigação deste trabalho, permite a representação e a comunicação de conceitos, ideias e de legado histórico. Mesmo que a percepção da arquitetura envolva atividades multissensoriais, a maneira dominante de concebê-la é visual. A representação virtual da arquitetura, é, portanto, importante fonte de conhecimento e veículo de informação (MÜNSTER et al., 2017). Paraizo (2010, p. 13) acrescenta que a representação do patrimônio, especialmente em meio digital oferece grande auxílio nesse sentido: "o computador, é em essência, uma ferramenta de manipulação simbólica. É na possibilidade de experimentação com os significados que reside sua maior contribuição".

Addison (2000), em paralelo, salienta que os rápidos avanços tecnológicos e as novas mídias trouxeram ao âmbito da herança cultural novas esperanças. As ferramentas e técnicas digitais presentes nos laboratórios de estudo, nos órgãos do governo e na indústria criativa passaram a dar um suporte cada vez maior às complexas atividades ligadas à arqueologia, à pesquisa histórica, à conservação dos monumentos e à educação patrimonial. Para esta prática dá-se o nome de patrimônio virtual (tradução do inglês virtual heritage).

0 conceito de patrimônio virtual, segundo Addison (2006, p. 2, tradução livre), consiste no "uso de tecnologias para registrar, modelar e visualizar o patrimônio cultural e natural". Suas ações compreendem um processo amplo, complexo e trabalhoso que vai desde a documentação do patrimônio estudado, à construção dos modelos tridimensionais (3D), passando pela disseminação e pelo uso/apropriação pelos usuários interessados. Vale frisar que o patrimônio virtual não se limita à tarefa modelagem 3D, como também o uso de novas ferramentas para documentação e registro do patrimônio, HBIM (Historic Building Information Modeling), ações que envolvam realidade aumentada, mista ou até mesmo a combinação delas (CANUTO; MOURA; SALGADO, 2017). A "virtualização do patrimônio", segundo Roussou (2002, p. 93), é onde reside a sua maior contribuição: a partir da computação gráfica, a prática do patrimônio virtual permite através de modelos virtuais, o registro, a simulação e visualização de artefatos culturais por meio de uma interface digital.

Alencar (2013) expõe que apesar de ser ainda incipiente, esta prática tem se mostrado cada vez mais difundida, e dentro do campo acadêmico, a virtualização computadorizada do patrimônio cultural é uma área de pesquisa relativamente recente e ainda que esteja em constante discussão, não foi alvo de debates mais consistentes. Fischer e Stinson (2007), por sua vez, explicam que o grande problema das reconstruções virtuais está no fato de que não há acordada, até o momento, uma linguagem da comunicação gráfica/visual no meio científico, o que torna inevitável o amadurecimento de conceitos e técnicas que orientem os trabalhos da área. Champion (2014) revela que, na tarefa de modelagem, muitos designers buscam níveis de detalhe que talvez nunca sejam percebidos pelos usuários, ou ainda mais, que podem ter interpretações diferentes pelo público final e para um especialista, por exemplo.

Neste sentido, este artigo é parte de uma pesquisa, desenvolvida no contexto do Programa de Pós-Graduação em Ambiente Construído (PROAC) da Universidade Federal de Juiz de Fora (UFJF) que busca investigar: (a) qual a influência do nível de detalhe (ou do inglês: Level of 
Detail - LOD) no processo (e nos resultados) de reconstrução virtual da Villa Ferreira Lage, importante edifício histórico localizado em Juiz de Fora, Minas Gerais, Brasil.

Para tanto, o trabalho descreve e analisa o processo de modelagem 3D da residência a partir da definição de cinco LODs, de maneira a comparar, para cada LOD adotado: (a) o tamanho dos arquivos 3D; (b) seus respectivos tempos de modelagem; (c) seus tempos de renderização, e; (d) a quantidade de dados dos modelos.

0 artigo trata uma pesquisa bibliográfica e de caráter experimental. Desta forma, está estruturado por meio da seguinte sequência: (i) uma breve revisão sobre patrimônio virtual; (ii) uma breve revisão sobre LOD e modelagem 3D de edifícios históricos; (iii) uma apresentação do método empregado; (iv) uma descrição do experimento desenvolvido; (v) uma apresentação e uma discussão sobre os resultados encontrados, e; (vi) uma discussão geral e considerações finais sobre a pesquisa em questão.

\section{PATRIMÔNIO VIRTUAL}

Champion e Dave (2007) afirmam que o início dos anos 1980 testemunhou os primeiros trabalhos que atrelaram computação gráfica às reconstruções históricas. Addison (2008) completa que a década de 1980 trouxe a popularização das ferramentas CAD (Computer Aided Design) e o advento do desenho digital, importante apoio às ações de documentação do patrimônio cultural. As primeiras discussões sobre as tecnologias de RV ocorreram a partir da década de 1990. Segundo Addison (2008), o pesquisador britânico Paul Reilley mencionou pela primeira vez, o termo 'arqueologia virtual' no seu texto para a conferência CAA (Computer Application in Archeology) e em 1998, 'patrimônio virtual' era o principal assunto das conferências anuais VSMM (Virtual System and MultiMedia). Desde então, as ferramentas digitais têm sido cada vez mais utilizadas em projetos focados em locais e contextos históricos por todo o mundo.

A primeira experiência de patrimônio virtual foi um tour virtual pelo Castelo de Dudley na Inglaterra, no ano de 1994 (JOHNSON, 1996 apud CHAMPION, 2014). Pouco tempo depois, o projeto Rome Reborn ${ }^{\circledR}$ começou a ser desenvolvido. De acordo com Alencar (2013), o trabalho é considerado um divisor de águas no contexto do patrimônio virtual. De autoria do CVRLab (Cultural Virtual Reality Lab) da Universidade da Califórnia, o projeto produziu modelos 3D acessíveis ao público em geral da cidade de Roma Antiga, desde a Idade do Bronze (cerca de 1.000 a.C.), até o seu despovoamento no início da Idade Média (aproximadamente em 500 d.C.).

Por outro lado, Champion (2014) afirma que os primeiros trabalhos de patrimônio virtual contavam com modelos dispersos, interação limitada e sobreposição excessiva de informações. A maioria dos trabalhos só podia ser acessada por telas de computadores e apresentavam aplicações pouco imersivas. Para Addison (2008), o aumento da qualidade dos projetos acompanhou a evolução dos métodos e ferramentas de documentação digital. A transferência de dados patrimoniais para o ambiente digital permitiu, ao longo dos últimos anos, o crescimento considerável dos níveis de qualidade, confiabilidade e interação.

Apesar do cenário parecer promissor e as ferramentas estarem em constante evolução, a construção dos modelos 3D de patrimônio virtual ainda enfrentam desafios. Sobretudo porque a disciplina possui produção científica incipiente, heterogênea e sem padrões estabelecidos. Segundo Pfarr-Harfst (2016), apesar da heterogeneidade e complexidade reconhecida no campo de estudo, ainda não foram desenvolvidas metodologias, diretrizes técnicas e padrões para os fluxos de trabalho. López-Menchero Bendicho et al. (2017) expõem que embora nos 
últimos anos, recomendações, princípios e organizações tenham sido concretizadas, o corpo teórico da disciplina ainda é pouco desenvolvido.

A partir das constatações expostas é coerente reconhecer a importância de se investigar os processos de modelagem 3D de arquiteturas históricas. Parâmetros como o LOD dos modelos, o tamanho e formato dos arquivos digitais, a infraestrutura para modelagem (hardware e software), o processamento dos dados e o nível de capacitação das equipes interferem diretamente no processo e nos resultados esperados (PFARR-HARFST, 2016).

Conforme López-Menchero Bendicho et al. (2017), atualmente dois documentos internacionais norteiam os projetos de patrimônio virtual: a Carta de Londres e a Carta de Sevilha. As cartas, apesar de não serem documentos publicados por órgãos oficiais de proteção do patrimônio cultural são importantes suportes para o desenvolvimento dos trabalhos da disciplina. Além disso, tais documentos não visam ao estabelecimento de objetivos e métodos específicos e sim, a princípios gerais para o uso das ferramentas digitais nos projetos.

0 primeiro rascunho da Carta de Londres foi publicado pelo King's Visualization Lab do King's College de Londres, em 2009 (LÓPEZ-MENCHERO BENDICHO et al., 2017). O documento estabelece seis princípios gerais para orientar o que ele chama de "visualização computadorizada do patrimônio cultural". Os princípios, em resumo, defendem o rigor científico dos trabalhos e a transparência do processo de construção dos modelos (i.e., paradados). A documentação do processo propõe que a linha de raciocínio por trás da compilação de todas as informações e agentes envolvidos seja divulgada. Isso permite que os métodos sejam avaliados e posteriormente utilizados em outros trabalhos. A Carta ainda defende que os métodos devem seguir os objetivos defnidos pela investigação e serem adaptados à metodologia traçada (e.g. mais ou menos fotorrealista, com maior ou menor LOD, mais geral ou mais preciso). A partir da definição destas questões, é possível melhorar os níveis de transparência científica e fornecer resultados de maior qualidade (DENARD, 2014).

A Carta de Sevilha foi publicada pela Sociedade Espanhola de Arqueologia Virtual, em 2012. Os princípios aprofundam as questões levantadas pela Carta de Londres e trazem uma abordagem voltada ao patrimônio arqueológico. Contudo, o documento apresenta importantes conceitos e explora outros olhares para a construção dos modelos de patrimônio virtual (LÓPEZMENCHERO BENDICHO et al., 2017). O documento conceitua a modelagem 3D de edifícios históricos como reconstrução virtual:

Reconstrução virtual: compreende o uso de um modelo virtual para recuperar visualmente, um dado momento de uma construção ou objeto feito por seres humanos no passado, a partir da evidência física existente dessa construção ou objeto, inferências comparativas cientificamente razoáveis e em geral todos os estudos realizados por arqueólogos e outros especialistas relacionados ao patrimônio arqueológico e à ciência histórica (IFVA, 2012, p. 3, tradução livre).

0 documento de Sevilha enumera oito princípios. Além de defender a transparência científica, sugere equipes interdisciplinares, autenticidade das informações e rigor histórico em todo o processo. Assim como na Carta de Londres, a Carta de Sevilha defende a documentação dos procedimentos realizados, a subordinação dos métodos aos objetivos traçados e que as a ferramentas digitais estejam sempre à serviço do patrimônio cultural, nunca o contrário. Os objetivos gerais de quaisquer trabalhos de patrimônio virtual devem considerar melhorias nos métodos de pesquisa, conservação e disseminação do patrimônio, bem como sempre servir à comunidade, de forma ampliar a sua conexão com os bens culturais (IFVA, 2012).

Se o objetivo dos trabalhos de patrimônio virtual é envolver pessoas e objetos virtuais para promover a salvaguarda de bens culturais, os projetos não devem focar somente no aspecto 
tecnológico, mas também nos aspectos filosóficos e criativos relacionados à representação dos modelos, à sua interação e avaliação (CHAMPION, 2014). Champion completa (2014, p. 6, tradução livre): o patrimônio virtual é a "tentativa de transmitir não apenas a aparência, mas também o significado dos artefatos culturais e da agência social associada a quem os projetou e os utilizou, através do uso de mídia digital interativa e imersiva". Porém, até que ponto seria desejável ou viável gerar modelos de alto LOD ou super realísticos? Qual a influência do LOD no processo e no resultado de modelos 3D de edifícios históricos? Estas são algumas das questões levantadas pelo estudo aqui proposto.

\section{NÍVEL DE DETALHE - LEVEL OF DETAIL - E MODELAGEM 3D DE EDIFÍCIOS HISTÓRICOS}

Para Heok e Daman (2004), a construção e disseminação de modelos virtuais exige tempo de trabalho e processamento gráfico. Buscando melhorar o desempenho dos processos e garantir qualidade gráfica, programadores passaram a usar a técnica de LOD para simplificar a geometria dos modelos 3D, mantendo sua qualidade de visualização e garantir armazenamento reduzido, menores taxas de processamento e rapidez na transferência de dados. Tolentino (2016), por sua vez, explica que o LOD é uma técnica que permite diferentes representações para um mesmo objeto, atuando diretamente na eficiência e no rendimento dos fluxos de trabalho.

De acordo com o BIMForum (2020), a sigla LOD é comumente confundida com nível de desenvolvimento (ou do inglês: Level of Development)i . Para tanto, é importante frisar que neste trabalho, LOD é utilizado para designar nível de detalhe. Ainda segundo o BIMForum (2020), nível de detalhe (LOD) diz respeito à quantidade de detalhe inserido na geometria do modelo, enquanto nível de desenvolvimento, trata em que grau a geometria dos elementos e as informações sobre eles foram anexadas ao modelo (i.e., inclui além do nível de detalhe, nível de informação, nível de precisão, dentre outros).

Graham, Chow e Fai (2019) discutem o fato de que, apesar de existirem diretrizes e padrões de modelagem para ferramentas BIM, tais padrões são voltados a novas construções, o que dificulta abordá-los em projetos que envolvam construções existentes e históricas. A modelagem 3D de edifícios históricos, portanto, deve avaliar os dados disponíveis sobre o bem, o propósito dos modelos 3D, assim como quais LODs são ideais e quais elementos devem ser mais detalhados (e.g. em uma aplicação digital que trate uma narrativa histórica, é importante identificar quais elementos têm valor patrimonial e qual o destaque deve ser dado frente à atenção dada pelos usuários).

Araujo et al. (2019) evidenciam a necessidade de se investigar sobre o LOD enquanto parâmetro de modelagem, no contexto do patrimônio virtual. Os autores realizaram um levantamento de 304 artigos sobre patrimônio virtual publicados entre 2013 e 2018 em eventos brasileiros e latino-americanos no qual os trabalhos foram divididos em grupos e classificados por suas palavras-chave. 0 grupo voltado às pesquisas que tratavam critérios de qualidade dos modelos 3D foi o que apresentou menor número de artigos. Os termos LOD (level of detail), LOI (level of information) e LOG (level of geometry) foram termos pouco encontrados, sobretudo em artigos brasileiros. Isso sugere que estudos voltados à interlocução entre LOD e modelagem/modelos 3D são um campo de pesquisa ainda pouco investigado no contexto brasileiro (ARAUJO et al., 2019).

A partir do entendimento de que a modelagem 3D tem como propósito a construção de modelos virtuais, é natural relacioná-la às tecnologias de realidade virtual. Para Lévy (2010), a 
realidade virtual é um tipo específico de simulação interativa onde o usuário tem a sensação de estar imerso em um ambiente construído virtualmente. "Ao manter uma interação sensóriomotora com o conteúdo de uma memória de computador, o explorador consegue a ilusão de uma "realidade" na qual estaria mergulhado: aquela que é descrita pela memória digital" (LÉVY, 2010, p. 74).

A simulação busca ampliar a capacidade de se estudar parâmetros de um modelo ${ }^{\text {ii }}$. Os modelos, portanto, nos permitem analisar diferentes hipóteses/contextos e avaliar o comportamento de cada um deles (LÉVY, 2011). No campo da pesquisa em patrimônio cultural, a RV permite a reconstrução de edifícios históricos, simula suas estruturas ao longo do tempo, oferece informações às diferentes atividades voltadas à preservação dos bens culturais e acima de tudo, amplia o acesso à informação. Porém o que é necessário para criar estes modelos 3D?

Pfarr-Harfst (2016) evidencia que os fluxos de trabalho e a metodologia de modelagem devem levar em consideração as ferramentas disponíveis e os objetivos traçados pelo projeto. 0 autor destaca que os procedimentos geralmente são realizados em 'mão única' e pouco integrados, o que torna o processo condicionado a uma série de dificuldades. A incompatibilidade entre os arquivos, as escalas de representação (i.e., os LODs) e a falta de compatibilidade entre as plataformas são citados como fatores que interferem no processo. No que tange aos edifícios históricos, o processo de modelagem encontra ainda maiores desafios (PFARR-HARFST, 2016).

Segundo Chevrier, Maillard e Perrin (2009), os edifícios históricos além de possuírem maior número de elementos arquitetônicos, apresentam também uma maior variedade de objetos a serem modelados. Fatores como o orçamento disponível, acesso à infraestrutura, disponibilidade de pessoal especializado, os objetivos do projeto e claro, a complexidade formal-geométrica do objeto de estudo são também condicionantes (QUINTERO; BLAKE; EPPICH, 2007).

Dentre todas as questões apresentadas, Pfarr-Harfst (2016) traz uma compilação de alguns pontos a serem considerados durante o processo de construção dos modelos 3D, são eles: (a) a escolha de um sistema técnico (hardware e software) condizente com os objetivos do trabalho; (b) a determinação de um LOD que possa potencializar os fluxos de trabalho; (c) a adoção de uma terminologia padrão que facilite o intercâmbio das informações; (d) a classificação e a estruturação das fontes consultadas; (e) a documentação correta e transparente do processo e; (f) a divulgação das etapas do fluxo de trabalho desde a coleta, processamento e compilação dos dados.

É possível reconhecer que a tarefa de modelagem em si requer conhecimento técnico, investimentos e, sobretudo, planejamento. 0 modelo 3D em projetos de patrimônio virtual é o que conecta as pessoas à sua herança cultural (i.e., um meio de comunicação) e, portanto, devese encontrar maneiras que potencializem o seu uso bem como facilitem a sua construção. 0 LOD, parâmetro aqui destacado, atua diretamente em como o objeto de estudo será representado e como a informação cultural chega até o usuário (MÜNSTER et al., 2017). É pensando na eficiência dos processos de modelagem 3D e na qualidade dos modelos 3D que se propõe investigar qual a influência dos LODs para a modelagem 3D do edifício escolhido.

\section{METODOLOGIA}

De acordo com Prodanov e Freitas (2013), os procedimentos definidos caracterizam esta pesquisa como bibliográfica e experimental, e que faz uso do método comparativo. 0 experimento definido apoia-se no mapeamento do processo de modelagem 3D a partir de cinco LODs, de forma a discutir a influência do LOD no tempo e processamento dedicado à 
modelagem 3D e nos resultados obtidos. 0 artigo, portanto, tem como foco principal o registro do processo de modelagem 3D da Villa Ferreira Lage e em como o edifício pode ser representado seguindo diferentes LODs.

Pensando nisso, os procedimentos foram divididos em seis principais etapas: (i) revisão de literatura sobre patrimônio virtual, cartas patrimoniais sobre o tema, LOD e modelagem 3D de edifícios históricos; (ii) pesquisa histórica sobre o edifício; (iii) tarefa de modelagem 3D em cinco diferentes LODs; (iv) análise comparativa dos dados quantitativos dos modelos; (v) discussão sobre os resultados e; (vi) conclusões sobre a relação entre o LOD e as representações obtidas da Villa Ferreira Lage.

O delineamento do experimento teve como referência os trabalhos de Münster et al. (2017) e Apollonio (2018). O trabalho de Münster et al. (2017) consiste em um experimento que relaciona LOD e percepção de formas arquitetônicas para um edifício localizado em Dresden, na Alemanha. Foram desenvolvidos seis LODs para representar uma edificação do fim do século XIX e, como resultado, os autores concluíram que altos níveis de abstração visual (i.e., menor LOD) e aparência esquemática são suficientes para tornar a arquitetura reconhecível para um observador. Contudo, o experimento de Münster et al. (2017) tem como objeto de estudo uma arquitetura de traços pouco rebuscados, e além disso, oriundo de uma realidade cultural europeia, a qual se distingue em diversos pontos do contexto brasileiro.

Apollonio (2018) ofereceu suporte à metodologia de modelagem 3D adotada - modelagem 3D por estruturas semânticas ou modelagem semântica. Seguindo o processo de modelagem baseado em fontes, o autor enumera as seguintes etapas: (i) coleta de dados documentais; (ii,a) análise das estruturas semânticas; (ii,b) análise das fontes (forma geométrica, aparência da superfície e características físicas - processo de análise por indução/dedução/analogia); (iii,a) enriquecimento semântico do modelo 3D; (iii,b) interligação entre os dados; (iv) modelagem 3D e; (v) validação da hipótese reconstrutiva (APOLLONIO, 2018, p. 140, tradução livre).

As estruturas semânticas são definidas por Apollonio (2018) como os elementos que compõem a "matriz formal-geométrica" da arquitetura. A metodologia usada para estruturar modelos semânticos se apoiam nos estudos da "gramática da forma" para elementos arquitetônicos (STINY; MITCHELL, 1978; STINY, 1980 apud APOLLONIO, 2018). A "gramática da forma" trabalha com diferentes níveis de abstração do espaço arquitetônico, permitindo identificar a lógica construtiva de um edifício e desenvolver um 'código de identidade' para cada elemento que posteriormente é elencado dentro de uma estrutura hierárquica (APOLLONIO, 2018).

\section{O EXPERIMENTO}

O objeto de estudo deste trabalho é a Villa Ferreira Lage, localizada no município de Juiz de Fora, Minas Gerais, Brasil. A residência teve a sua construção encomendada pelo comendador Mariano Procópio Ferreira Lage (1821-1872) para receber Dom Pedro II (1825-1891) na inauguração da estrada "União Indústria" em 1861. Contudo, o edifício só foi visitado pelo imperador em 1869. Atualmente ele é um dos mais importantes elementos arquitetônicos do Conjunto Arquitetônico e Paisagístico do Museu Mariano Procópio, resultado do hábito colecionista de Alfredo Ferreira Lage (1895-1944) - filho mais novo do comendador Mariano Procópio - que reuniu durante a vida um dos mais significativos acervos artísticos, históricos e de ciências naturais do Brasil. Foi aberto à visitação como museu particular em 1915, e inaugurado oficialmente em 23 de junho de 1921 (FASOLATO, 2014).

0 edifício possui estilo arquitetônico de inspiração renascentista e seu projeto é de autoria do arquiteto alemão Carl August Gambs. A casa foi implantada no alto do terreno em meio a um 
parque, distribuída em dois pavimentos, um torreão e um porão (FASOLATO, 2014). Sua proteção legal já alcança as três esferas: municipal (2005), estadual (2005) e federal (2015).

A modelagem 3Diii do edifício foi desenvolvida no software SketchUp da Trimble $\subset$ e, conforme já comentado, partiu da definição de cinco LODs. Segundo Biljecki, Ledoux e Stoter (2016), pelo fato do LOD ser um parâmetro que afeta a representação do objeto, ele pode ser adaptado à demandas específicas, ou seja, não possui regras pré-definidas. Neste trabalho, os LODs foram atrelados ao número de estruturas semânticas presentes no modelo 3D. Abaixo segue a relação de quais estruturas semânticas cada LOD compreende:

- LOD01: volumetria;

- LOD02: volumetria + cobertura + aberturas;

- LOD03 - 'LOD controle': volumetria texturizada (geometria atrelada às ortofotos);

- LOD04: volumetria + aberturas + esquadrias + elementos decorativos;

- LOD05: volumetria + aberturas + esquadrias + elementos decorativos + materiais de acabamento.

Figura 1. Esquema ilustrativo da modelagem 3D do edifício e os cinco LODs definidos.

Fonte: Dos autores.

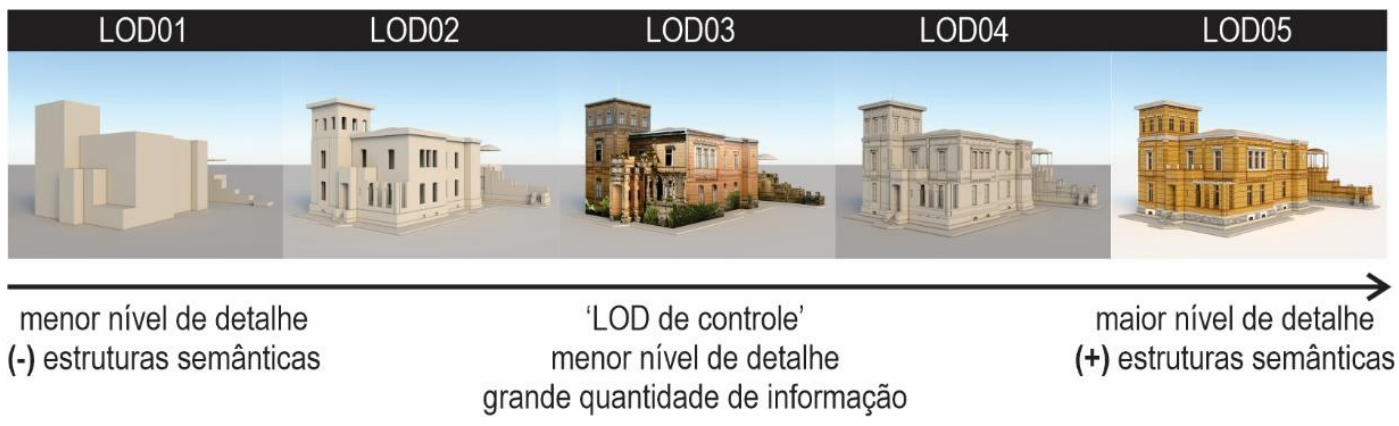

O LOD03 foi definido como 'LOD de controle', uma vez que apresenta alta quantidade de informação visualiv em um modelo 3D com baixo LOD. A técnica utilizada se difere da utilizada para os demais LODs: para o LOD03, as fachadas do edifício foram texturizadas por ortofotos. Segundo Bastian (2015), as ortofotos são fotografias convencionais que passam por transformações para que sejam retificadas e as distorções de perspectiva eliminadas. Como resultado, tem-se um modelo 3D que permite representar a arquitetura investigada sem maiores trabalhos de modelagem. Pode-se dizer que as texturas 'substituem' a modelagem dos detalhes construtivos e dos elementos decorativos do edifício, criando-se uma falsa impressão perceptual.

A partir da pesquisa histórica e do levantamento métrico em AutoCAD fornecido pela Fundação Museu Mariano Procópio (MAPRO), foram modeladas as quatro fachadas do edifício e somente a divisão interna dos ambientes. Os desenhos de levantamento foram alinhados ortogonalmente ao modelo e utilizados como referência para posicionar os elementos em geral. A Figura 3 ilustra o processo de modelagem 3D da Villa Ferreira Lage, que seguiu três etapas principais. 


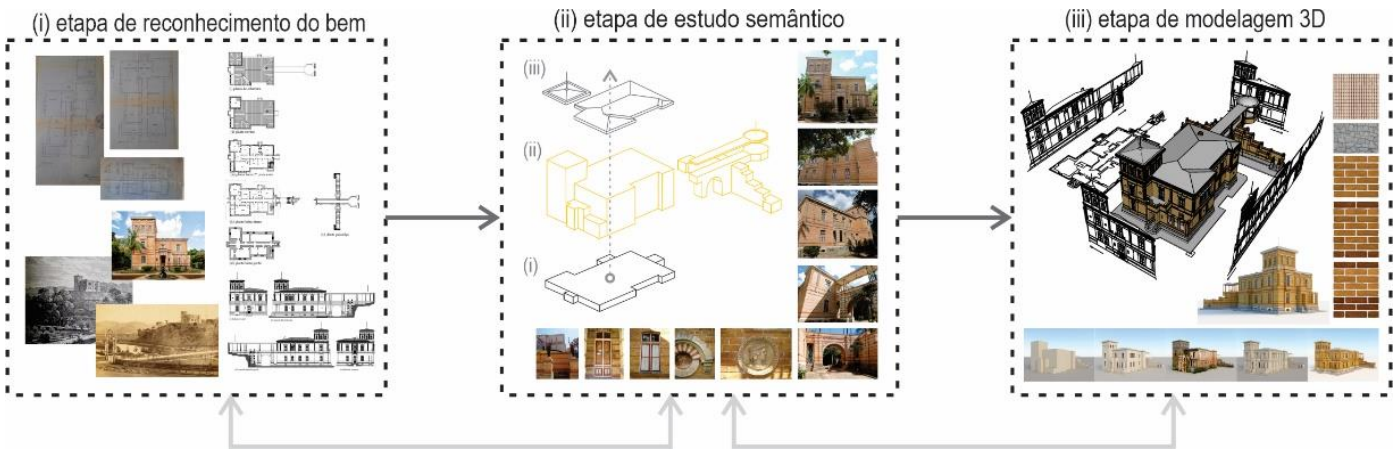

A (i) etapa de reconhecimento do bem foi destinada à coleta de dados documentais sobre o objeto de estudo. Contou, portanto, com (i-a) a visita ao Acervo Histórico do Museu Mariano Procópio; (i-b) o acesso à documentação histórica e digital da Villa Ferreira Lage; (i-c) a análise de fotografias provenientes de acervo pessoal e da web e com; (i-d) a análise de textos e publicações científicas sobre a arquitetura da Villa Ferreira Lage. A (ii) etapa de estudo semântico foi baseada na metodologia exposta por Apollonio (2018), partindo da análise geométrica e construtiva do edifício. A partir da (ii-a) criação de um esquema formalgeométrico do edifício (edifício foi 'dividido' em embasamento, corpo e cobertura); (ii-b) os elementos arquitetônicos e suas respectivas hierarquias foram identificadas e organizadas para; (ii-c) a delimitação dos cincos LODs.

Enquanto, a (iii) etapa de modelagem 3D partiu da tarefa de (iii-a) importar os desenhos em AutoCAD para o SketchUp; (iii-b) criação dos planos de contorno do edifício (geometria base) para posterior modelagem 3D em dois níveis: (iii-c) modelagem 3D 'vertical' e 'de baixo para cima' (extrusão dos sólidos primários da geometria do edifício) e (iii-d) modelagem 3D 'horizontal' ou enriquecimento semântico do modelo (alocação dos detalhes construtivos e arquitetônicos). Por fim, (iii-e) os elementos arquitetônicos foram diferenciados (i.e., separados em grupo e/ou componentes); (iii-f) os modelos texturizados com mapas criados a partir de fotografias ou provenientes de repositórios online para que fossem (iii-g) renderizados e (iii-h) apresentados.

Os modelos foram renderizados e as imagens ilustrativas geradas (com resolução 3.000X2.252 pixels e sem nenhum processo de pós-produção). Os materiais de acabamento não receberam configurações de refletância e/ou rugosidade. Os modelos 3D passaram somente pelo mapeamento de texturas, uma vez que o objetivo é investigar o processo de modelagem 3D, excluindo, portanto, o nível de fotorrealismo das imagens ou a qualidade puramente gráfica das representações.

Durante o processo, foram documentados quatro dados referentes à modelagem $3 \mathrm{D}$ de cada LOD. São eles: (a) o tamanho do arquivo (TA); (b) o tempo de modelagem (TM); (c) o tempo de renderização (TR) e; (d) a quantidade de dados do modelo (QDM). Os dados foram obtidos por anotações mediante a execução de cada etapa e posteriormente comparados de maneira que o volume de trabalho e as exigências de processamento da máquinav ${ }^{\mathrm{v}}$ pudessem ser mensurados nas diferentes situações.

\section{RESULTADOS E DISCUSSÃO}

0 processo de modelagem encontrou algumas facilidades e dificuldades, conforme apresentado a seguir. 0 planejamento das etapas de modelagem (PFARR-HARFST, 2016) e a técnica de modelagem por enriquecimento semântico do modelo (APOLLONIO, 2018) trouxeram grandes ganhos de tempo, pois quando a lógica do edifício é compreendida, a
Figura 2. Etapas do processo de modelagem $3 D$ da Villa Ferreira Lage.

Fonte:

Dos autores. 
organização dos seus elementos arquitetônicos passa a ser facilitada. Outro ponto a ser destacado foi a transformação dos elementos decorativos em componentes vi. Essa opção trouxe automatização à modelagem 3D. As configurações dadas a um componente são replicadas aos seus similares. Já os elementos únicos (i.e., volumetria, cobertura, cornijas, escadarias) foram transformados em grupos. Chevrier, Maillard e Perrin (2009) afirmam que a criação de "formas elementares" permite a criação de modelos paramétricos e estes, tornam a modelagem mais rápida. O LOD04 e LOD05 apresentaram 33 componentes, ou seja, 33 "formas elementares".

As dificuldades encontradas também vão de acordo com o exposto por Chevrier, Maillard e Perrin (2009). A Villa Ferreira Lage é uma edificação com grande quantidade e variedade de elementos decorativos e isto afetou de forma direta o tempo de modelagem. Além disso, o edifício não possui contornos ortogonais. A falta de ângulos retos na geometria do edifício também dificultou a modelagem dos elementos e a aplicação de texturas. Ao longo do processo, a conferência de medidas e a consulta aos registros fotográficos foram essenciais para a modelagem 3D do edifício.

Além destas questões levantadas, é também importante frisar que os modelos 3D de edifícios históricos não seguem os métodos 'convencionais' de modelagem 3D. Uma modelagem 'convencional' tem como fonte de informações as representações do objeto, uma vez que ele existe somente no campo das ideias. Objetos existentes têm duas fontes de informação: o próprio objeto físico e a sua documentação (i.e., levantamentos métricos, fotográficos, relatos, etc.). A modelagem 3D de edifícios históricos segue o tipo de modelagem por engenharia reversa (tradução do inglês reverse engineering ou back engineering) (KOMMULA et al., 2018).

0 processo de modelagem 3D, no total, levou 35 horas. As tarefas foram executadas durante 16 dias não consecutivos e seguindo as etapas delineadas. 0 tempo dedicado ao estudo semântico do edifício foi incluído na etapa de construção do LOD01. Vale lembrar que esta contagem é referente ao processo de modelagem 3D e exclui as demais etapas de trabalho (e.g., visitas ao local e ao acervo). A Tabela 1 quantifica todos os dados coletados para cada um dos cinco LODs.

Tabela 1. Relação dos dados obtidos para os cinco LODs definidos no modelo 3D da Villa Ferreira Lage.

Fonte: Dos autores.

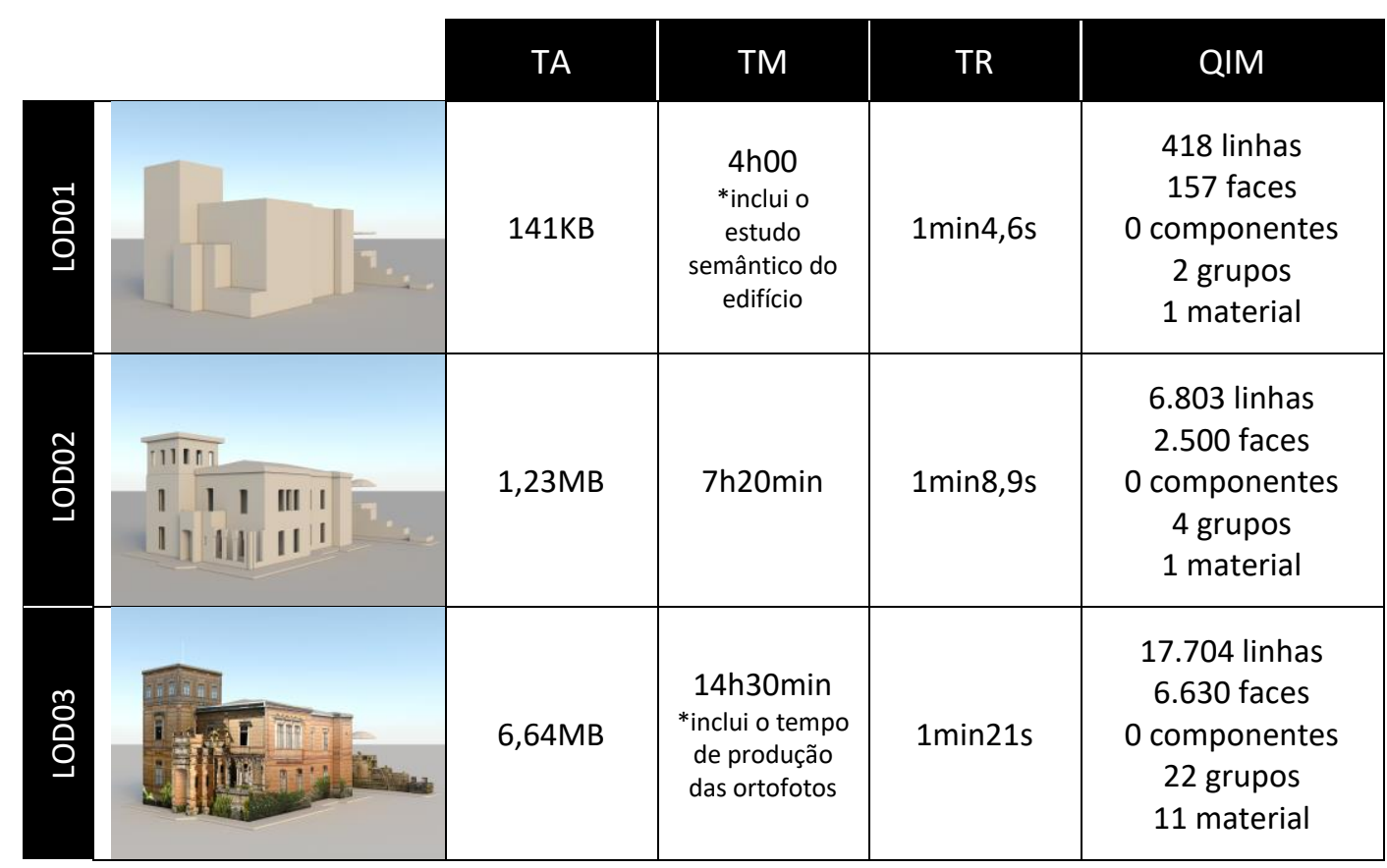




\begin{tabular}{|c|c|c|c|c|}
\hline 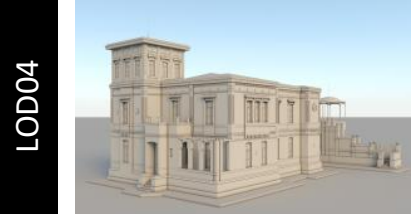 & $32,80 \mathrm{MB}$ & 27h30min & $2 \mathrm{~min} 40 \mathrm{~s}$ & $\begin{array}{c}3.462 .171 \text { linhas } \\
1.845 .252 \text { faces } \\
33 \text { componentes } \\
651 \text { grupos } \\
1 \text { material }\end{array}$ \\
\hline  & $34,40 \mathrm{MB}$ & $35 \mathrm{~h} 00$ & $4 \min 50 s$ & $\begin{array}{c}3.462 .171 \text { linhas } \\
1.845 .252 \text { faces } \\
33 \text { componentes } \\
651 \text { grupos } \\
12 \text { material }\end{array}$ \\
\hline
\end{tabular}

O tamanho dos arquivos (TA) apresentou grande variação. Na medida em que a geometria do edifício é modificada e maior número de objetos geométricos e texturas são inseridos, os arquivos tornam-se mais robustos. Pfarr-Harfst (2016) atenta sobre a importância na escolha do sistema técnico, que, neste caso, deve prever a capacidade de armazenamento do hardware da máquina utilizada. 0 tamanho do arquivo afeta o armazenamento dos arquivos, o seu compartilhamento e o processamento da máquina, influenciando no rendimento e na duração das etapas de modelagem.

Como o esperado, a modelagem 3D das representações com maior LOD exigiu maior tempo de trabalho. 0 tempo de modelagem (TM) apresentou o seguinte comportamento: TMLOD01<TM-LOD02<TM-LOD03<TM-LOD04<TM-LOD05. 0 tempo de modelagem, portanto, esteve diretamente ligado à riqueza de detalhes do modelo. Quanto maior o LOD, maior o tempo de modelagem. Esta constatação, além de óbvia, vai ao encontro do exposto por PfarrHarfst (2016): os fluxos de trabalho devem definir um sistema técnico que seja coerente com a representação necessária e os LODs devem ser reflexo dos objetivos e do design a ser utilizado.

O tempo de renderização (TR) não apresentou altos valores. A relação que pode ser feita é que quanto maior o LOD, mais elaborada a geometria e maior quantidade de texturas aplicadas ao modelo 3D, maior é o tempo de renderização. Se considerarmos que o LOD03 e LOD05 apresentam modelos com quantidade de informação visual próximas (i.e., apresentam elementos com texturas e materiais de acabamento), pode-se afirmar que os tempos de renderização apresentaram variação considerável - TR-LOD03 é cerca de quatro vezes menor que TR-LOD05.

Por fim, mediu-se a quantidade de dados do modelo (QDM). Foram mensurados cinco itens: (i) número de linhas; (ii) faces; (iii) componentes; (iv) grupos, e; (v) materiais de cada modelo. 0 número de materiais representa a quantidade de texturas existentes nos mesmos. Estes parâmetros dizem respeito às estruturas geométricas e de acabamento do modelo 3D. Os dados de QDM foram obtidos de forma automática pelo software de modelagem. 0 valor de QIM referente a cada LOD é produto da soma dos cincos parâmetros, uma vez que eles foram considerados como elementos que fornecem informação visual ao modelo 3D. 
Figura 3. Variação dos dados medidos para os cinco LODs definidos no modelo 3D da Villa Ferreira Lage. Fonte:
Dos autores.

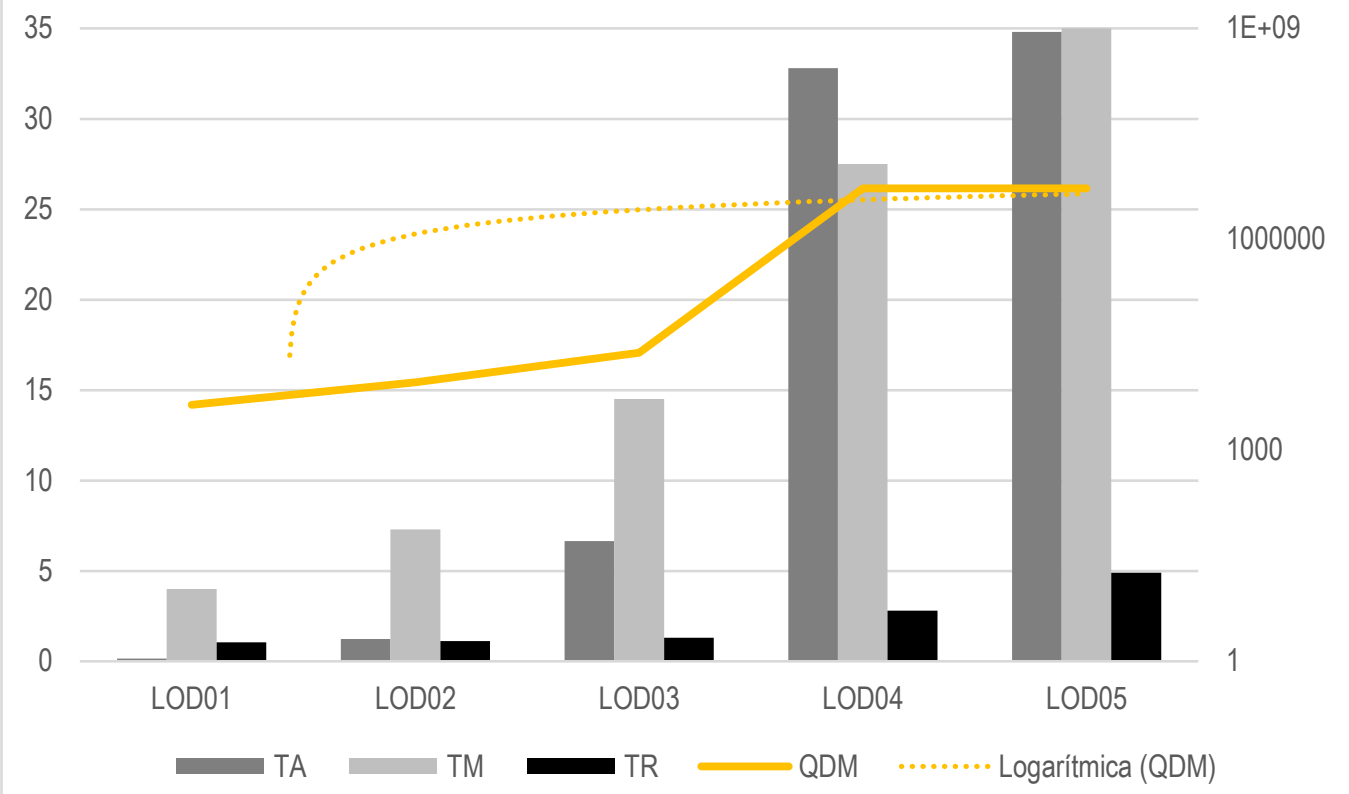

De acordo com o gráfico contido na Figura 3, é possível constatar que a diferença entre os parâmetros medidos pelo QDM-LOD01 é grande em relação ao QDM-LOD05. A "Logarítmica (QDM)" é uma linha de tendência em escala logarítmica. Ela determina a taxa de variação entre os dados medidos. Se analisarmos o comportamento da linha de tendência é possível perceber que a curva tem inclinação mais acentuada entre os valores de QDM-LOD01 e QDM-LOD-02, contudo, passa a se estabilizar a partir do LOD03. Os valores de QDM-LOD04 e QDM-LOD05 apresentaram pouca variação. Isto se deve ao fato de que a diferença entre o LOD04 e LOD05 está somente na quantidade de materiais aplicados à geometria, cujo número é pequeno, porém com distintos níveis de informação visual.

\section{CONSIDERAÇÕES FINAIS}

Neste cenário, pode-se concluir que a pesquisa e os projetos de patrimônio virtual possibilitam maior difusão do patrimônio arquitetônico, pois torna-o passível de experimentação mesmo que de maneira remota e virtual. 0 processo de reconstrução virtual requer o levantamento de dados sobre o edifício e a sua respectiva transferência para o ambiente virtual. A modelagem 3D de edifícios históricos é considerada, portanto, uma importante ferramenta neste sentido, uma vez que permite a construção destes espaços. 0 planejamento das atividades de modelagem e a modelagem 3D por estruturas semânticas atrelada aos cinco LODs definidos trouxeram ganhos de tempo para os processos aqui realizados.

O mapeamento do processo de modelagem 3D permitiu entender e comparar a quantidade de tempo dedicada na construção dos cinco modelos. Acredita-se que o mapeamento dos fluxos de trabalho possa aprimorar os processos de modelagem 3D e claro, garantir maior qualidade e rigor científico dos modelos. Ainda que não existam muitas investigações neste sentido, acredita-se que elas podem oferecer aos designers maior suporte ao planejar o tempo a ser dedicado na modelagem e alinhá-lo ao resultado final dos modelos 3D. Este artigo focou justamente nas questões que influenciam o planejamento e o rendimento das tarefas de modelagem. 
Da mesma forma que é preciso entender que as metodologias de modelagem 3D para edifícios históricos não seguem métodos 'convencionais' de modelagem, é necessário que o designer tenha experiência profissional e conhecimento técnico arquitetônico sobre a edificação que se pretender modelar. 0 domínio dos softwares de modelagem, assim como de técnicas de manipulação de desenhos digitais, edição de fotografias e noções sobre renderização de modelos 3D também foram essenciais para a produção dos modelos aqui apresentados.

0 experimento realizado demonstrou relação direta entre os fluxos de trabalho de modelagem 3D e os LODs adotados. À medida em que os modelos passaram a possuir maior riqueza de detalhes, o tamanho dos arquivos (TA) aumentaram, o tempo de modelagem (TM) e tempo de renderização (TR) foram afetados e a quantidade de dados do modelo (QDM) alcançou grandes variações. Contudo, se compararmos o LOD03 e o LOD05, podemos perceber que os modelos apresentam grande quantidade de informação visual, apesar de passarem por processos de modelagem com grandes diferenças.

Os resultados obtidos ainda mais uma questão: dedicar maior tempo e maior processamento de máquina para a execução do LOD05 em contrapartida com o LOD03 influencia até que ponto a percepção do edifício? Até que ponto uma representação esquemática de um edifício histórico torna a sua compreensão facilitada? Estas questões serão tratadas em trabalhos futuros decorrentes desta pesquisa. Do exposto, pretende-se investigar o nível de percepção das pessoas (NPP) para cada um dos LODs definidos, comparando-os com o edifício físico. Desta forma, acredita-se ser possível oferecer respostas mais assertivas sobre a relação entre LOD, processo de modelagem 3D e a disseminação de modelos 3D de edifícios históricos.

\section{Referências Bibliográficas}

ADDISON, A. C. Emerging Trends in Virtual Heritage. IEEE Multimedia, v.7, n.2, p. 22-25, apr./jun. 2000. DOI: $10.1109 / 93.848421$.

ADDISON, A. C. The Vanishing Virtual: Safeguarding heritage's endangered digital record. In: KALAY, Y.; KVAN, T.; AFFLECK, J. (Eds.). New Heritage: New Media and Cultural Heritage. Nova York: Routledge, p. 27-39, 2006. DOI: https://doi.org/10.4324/9780203937884.

ADDISON, A. C. Digital Heritage 2.0: Strategies for Safeguarding Culture in a Disappearing World. In: Proceedings of International Symposium on "Information and Communication Technologies in Cultural Heritage", loannina, 2008. Disponível em:

$<$ http://www.academia.edu/2519668/Digital_Heritage_2.0_Strategies_for_Safeguarding_Culture _in_a_Disappearing_World> Acesso em: 20 ago. 2020.

ALENCAR, A. T. S. A gráfica digital e o patrimônio arquitetônico - panorama e estado da arte. In: XXI Simpósio Nacional de Geometria Descritiva e Desenho Técnico, X International Conference on Graphics Engineering for Artes and Design, de 03 a 05 de novembro de 2013: Tecnologia e Arte para Inovação. Anais... Florianópolis: Editora CCE - UFSC, 2013.

APOLLONIO, FI. The Production of 3D Digital Archives and the Methodologies for Digitally Supporting Research in Architectural and Urban Cultural Heritage. In: MÜNSTER, et al. (Eds.). Digital Research and Education in Architectural Heritage, p. 139-158, 2018. Springer. DOI: https://doi.org/10.1007/978-3-319-76992-9_9.

ARAUJO, A. P. R.; CARLOS, C. A. S. L.; SAMPAIO, J. C. R.; VIEIRA, R. F. Digital heritage: academic research in Brazil in last five years. In: The International Archives of Photogrammetry, Remote Sensing and Spatial Information Sciences, Volume XLII-2/W15, 2019; 27th CIPA International 
Symposium "Documenting the past for a better future", 1-5 September 2019, Ávila, Spain. DOI: https://doi.org/10.5194/isprs-archives-XLII-2-W15-109-2019.

BASTIAN, A. V. Métodos e técnicas de baixo custo para levantamento métrico de sítios históricos. 2015. Dissertação (Mestrado em Arquitetura e Urbanismo) - Programa de PósGraduação em Arquitetura e Urbanismo, Universidade Federal da Bahia, Salvador, 2015.

BILJECKI, F.; LEDOUX, H.; STOTER, J. An improved LOD specification for 3D building models. Computers, Environment, and Urban Systems, vol. 59, p. 25-37, 2016.

http://doi.org/10.1016/j.compenvurbsys.2016.04.005.

BIMForum. BIM Forum Level of Development (LOD) Specification 2020. 2020. Disponível em: $<$ www.bimforum.org/lod > Acesso em: 20 jan. 2021.

CHAMPION, E. History and Cultural Heritage in Virtual Environments. In: GRIMSHAW, M. (Ed.), The Oxford Handbook of Virtuality. Oxford: Oxford University Press, p. 269-283, 2014. DOI:10.1093/oxfordhb/9780199826162.013.020.

CHAMPION, E.; DAVE, B. Dialing up the past. In: CAMERON, F.; KENDERDINE, S. (Eds.). Theorizing Digital Cultural Heritage: A Critical Discourse, (1). Cambridge: The MIT Press, p. 333-347, 2007. ISBN 978-0-262-03353-4.

CHEVRIER, C.; MAILLARD, Y.; PERRIN, J. P. A method for the 3d modelling of historic monuments: the case of a gothic abbey. In: Proceedings of ISPRS Workshop 3D ARCH, International Archives of Photogrammetry, Remote Sensing and Spatial Information Sciences. 2009. Disponível em: <http://citeseerx.ist.psu.edu/viewdoc/download?doi=10.1.1.363.3557\&rep=rep1\&type=pdf > Acesso em: 20 ago. 2020.

DENARD, H. (Ed.). Carta de Londres para a Visualização Computadorizada do Património Cultural (versão 2.1). Tradução de Maria Leonor Botelho e Ricardo M. Dias. 1 de Setembro de 2014. Disponível em: <http://www.londoncharter.org/fileadmin/templates/main/docs/london_charter_2_1_pt.pdf > Acesso em: 20 ago. 2020.

FASOLATO, D. Museu Mariano Procópio: a casa do pai, a casa do filho. In: PESSOA, A.; RANGEL, A. (Orgs.). III Encontro Luso-Brasileiro de Museus Casas: espaço, memória e representação. Rio de Janeiro, de 10 a 13 de agosto de 2010. Anais... Rio de Janeiro: Fundação Casa de Rui Barbosa, p. 34-46, 2014.

FISCHER, B.; STINSON, P. The importance of scientific authentication and a formal visual language in virtual models of archaeological sites: the case of the house of Augustus and Villa of the Mysteries. In: CALLEBAUT, D.; N. A. SILBERMAN (Eds.); Interpreting the Past: Heritage, New Technologies and Local Development, Proceendings... Bruxelas: Flemish Heritage Institute, p. 4983, 2007.

GOMES FILHO, J. Gestalt do objeto: sistema de leitura visual da forma. 8 ed. São Paulo: Escrituras Editora, 2008.

GRAHAM, K.; CHOW, L.; FAI, S. From BIM to VR: defining a level of detail to guide virtual reality narratives. Journal of Information Technology in Constrution (ITCon), v.24, p. 553-568. 2019. DOI: $10.36680 /$ j.itcon.2019.031.

HEOK, T. K.; DAMAN, D. A Review on Level of Detail. In: International Conference on Computer Graphics, Imaging and Visualization (CGIV'04), 26-29 July, 2004. Proceedings... Penang: Malásia, p. 70-75, 2004. DOI: 10.1109/CGIV.2004.1323963. 
IFVA. International Forum of Vitual Archeoloy. Principles of Seville. International Principles of Virtual Archeology. 2012. Disponível em: < http://smartheritage.com/seville-principles/sevilleprinciples> Acesso em: 20 ago. 2020.

KOMMULA, K. B.; LAUDYA, S. T.; ESLAVATH, S.; KARRI, S. N. R.; PANDEY, R. Reverse Engineering for Restoration and Preservation of Old Artifacts and Cultural Heritage. International Journal of Engineering Research in Mechanical and Civil Engineering (IJERMCE), v.3, n.3, Mar. 2018. ISSN (Online) 2456-1290.

LÉVY. P. Cibercultura. 3 ed. Tradução de Carlos Irineu da Costa. São Paulo: Editora 34, 2010.

LÉVY. P. O que é virtual? 2 ed. Tradução de Paulo Neves. São Paulo: Editora 34, 2011.

LÓPEZ-MENCHERO BENDICHO, V.; GUTIÉRREZ, M. F.; VINCENT, M. L.; LEÓN, A. G. Digital Heritage and Virtual Archaeology: and Principles of 3D Digital Reconstruction of Cultural Heritage. In: MÜNSTER, et al. (Eds.). Mixed Reality and Gamification of Cultural Heritage. Springer, p. 3-26, 2017. DOI: 10.1007/978-3-319-49607-8_1.

MÜNSTER, S.; KRÖBER, C; WELLER, H.; PRECHTEL, N. Virtual Reconstruction of Historical Architecture as Media as Knowledge Representation. In: IOANNNIDES, et al. (Eds.). Mixed Reality and Gamification of Cultural Heritage. Springer, p. 313-330, 2017. DOI 10.1007/978-3-319-496078_12.

PARAIZO, R. C. Representação do espaço urbano significado em interfaces digitais. In: MACHADO, Denise P. et al. (Org.). I Encontro Nacional da Associação Nacional de Pesquisa e Pós-Graduação em Arquitetura e Urbanismo. Anais... Rio de Janeiro: PROURB, 2010.

PFARR-HARFST, M. Typical Workflows, Documentation Approaches: An Approach Through the Framework of International Recommendations. In: MÜNSTER, et al. (Eds.). 3D Research Challenges in Cultural Heritage II. Springer, p. 32-46, 2016. DOI: 10.1007/978-3-319-47647-6_2.

PRODANOV, C. C.; FREITAS, E. C. Metodologia do trabalho científico: métodos e técnicas de pesquisa e do trabalho acadêmico. 2 ed. Novo Hamburgo: Feevale, 2013.

QUINTERO, M. S.; BLAKE, B.; EPPICH, R. Conservation of Architectural Heritage: The Role of Digital Documentation Tools: The Need of Appropriate Teaching Material. International Journal of Architectural Computing, v.5, n.2, p. 239-252, 2007. DOI: 10.1260/1478-0771.5.2.240.

ROUSSOU, M. Virtual Heritage: From the Research Lab to the Broad Public. In: NICCOLUCCI, F. (Ed.) Virtual Archeology: Proceedings of the VAST Euroconference, 24-25 November 2000; BAR International Series. Arezzo: Italy, Archeopress Oxford, p. 93-100. 2002. ISBN: 1841714542.

TOLENTINO, M. M. A. A utilização do HBIM na documentação, gestão e na preservação do Patrimônio Arquitetônico. In: SIGraDi 2016, XX Congress of the Iberoamerican Society of Digital Graphics, 9-11 November, 2016. Proceedings... Buenos Aires: Argentina, p. 510-518, 2016. DOI: $10.5151 /$ despro-sigradi2016-534.

\section{Notas}

João Paulo Chagas Maia Vilela joao.vilela@arquitetura.ufif.br

Ricardo Ferreira Lopes ricardo.lopes@arquitetura.ufjf.br

Fernando Lima fernando.lima@arquitetura.ufjf.br

\footnotetext{
' A BIMForum é uma instituição norte americana que propôs, em 2013, o "Level of Development Specification" cuja função é criar uma ferramenta de referência que visa melhorar a qualidade de
} 
comunicação e das características dos elementos dos modelos de usuários do BIM. A sigla LOD, para o BIMForum (ibid.), refere-se a nível de desenvolvimento.

ii Para Lévy (2011, p. 68), os modelos são descrições rigorosas de objetos ou fenômenos que serão posteriormente simulados.

iii A modelagem 3D foi executada pelo primeiro autor do artigo cuja formação é em Arquitetura e Urbanismo e possui experiência de seis anos com modelagem 3D como estagiário, arquiteto e tutor da disciplina de "Modelagem digital técnica para Arquitetura e Urbanismo I" da Faculdade de Arquitetura e Urbanismo da UFJF (FAU-UFJF) no período de um semestre letivo.

iv Para Gomes Filho (2008, p. 25), a informação visual pode ser entendida como "qualquer coisa visível, qualquer manifestação visual concreta passível de ser lida, analisada e interpretável formalmente".

$\vee$ O computador utilizado para a modelagem 3D do edifício possui a seguinte configuração: processamento Intel ${ }^{\circledR}$ Core $^{\text {TM }}$ i7-9750H, memória (RAM) de $16 \mathrm{~GB}$ e placa de vídeo NVIDIA ${ }^{\circledR}$ GTX $^{\mathrm{TM}}$ 1660Ti de 6GB de memória dedicada.

vi A técnica de criação de componentes e grupos foi possível pelo software de modelagem utilizado e desta forma, vale ressaltar que os resultados obtidos se validam para este contexto em específico. 